\title{
Gestational weight gain and offspring's cognitive skills: a systematic review and meta-analysis
}

Jose Alberto Martínez-Hortelano ${ }^{1}$, Celia Álvarez-Bueno ${ }^{1,2}$, Iván Cavero-Redondo 1,2*, Ángel Herráiz-Adillo ${ }^{1}$, Carlos Berlanga-Macías ${ }^{1}$ and Vicente Martínez-Vizcaíno ${ }^{1,3}$

\begin{abstract}
Background: Gestational weight gain has been associated with some adverse perinatal outcomes, but few studies have examined the association between gestational weight gain and offspring's cognition and their conclusions are inconsistent. Our systematic review and meta-analysis aimed to synthesize the evidence regarding the association between gestational weight gain and offspring's cognitive skills.

Methods: In this systematic review and meta-analysis (PROSPERO number, CRD42017073266), we systematically searched MEDLINE, EMBASE, Web of Science and the Cochrane Library for studies examining association between gestational weight gain and offspring's cognitive skills, without restriction in study design or language. Two reviewers extracted in an independent way the data. The Quality of Reporting of Observational Longitudinal Research scale was used to assess the quality of included studies. Effect size (ES) for adjusted models and their corresponding 95\% confidence intervals were calculated for (i) intelligence quotient, (ii) language related skills and (iii) mathematic related skills comparing offspring's cognitive skills when gestational weight gain was within recommendations (as reference) with those from mothers whose gestational weight gain was above or below the recommendations.

Results: Thirteen studies were included. There was a positive trend that associated gestational weight gain above recommendations with better offspring's intelligence quotient, although not statistically significant (ES $0.02,95 \% \mathrm{Cl}$ $\left.-0.00,0.05 ; 1^{2}=0.00 \%\right)$.

Conclusions: There is a not significant positive association between gestational weight gain above recommendations and intelligence quotient and some studies reported associations between gestational weight gain and offspring's cognitive skills. Our analyses confirm a wide variability in the results of studies published so far and highlights the need for conducting studies including specific samples of pregnant women by pre-pregnancy body mass index and trimester of pregnancy.
\end{abstract}

Keywords: Pregnancy, Gestational, Weight gain, Cognition, Children

\footnotetext{
* Correspondence: Ivan.Cavero@uclm.es

'Universidad de Castilla-La Mancha, Centro de Estudios Sociosanitarios,

Cuenca, Spain

2Universidad Politécnica y Artística del Paraguay, Asunción, Paraguay

Full list of author information is available at the end of the article
}

(c) The Author(s). 2020 Open Access This article is licensed under a Creative Commons Attribution 4.0 International License, which permits use, sharing, adaptation, distribution and reproduction in any medium or format, as long as you give appropriate credit to the original author(s) and the source, provide a link to the Creative Commons licence, and indicate if changes were made. The images or other third party material in this article are included in the article's Creative Commons licence, unless indicated otherwise in a credit line to the material. If material is not included in the article's Creative Commons licence and your intended use is not permitted by statutory regulation or exceeds the permitted use, you will need to obtain permission directly from the copyright holder. To view a copy of this licence, visit http://creativecommons.org/licenses/by/4.0/ The Creative Commons Public Domain Dedication waiver (http://creativecommons.org/publicdomain/zero/1.0/) applies to the data made available in this article, unless otherwise stated in a credit line to the data. 


\section{Background}

Recommendations for gestational weight gain (GWG) have been a debatable issue during the last three decades. In 2009, the Institute of Medicine (IOM) updated GWG recommendations published in 1990 by The National Academy of Science [1], changing pre-gestational body mass index (BMI) classification and suggesting less GWG for obese women to improve perinatal outcomes [2]. Nowadays, according to the IOM recommendations in developed countries, 42.9 and $20 \%$ of pregnant women had excessive or insufficient GWG, respectively, making it a growing public health problem [3-5].

Excessive GWG increases the risk of some adverse perinatal outcomes, such as caesarean delivery, hypertensive disorders of pregnancy, macrosomia, neonatal hypoglycaemia or shoulder dystocia [6]. Furthermore, excessive GWG has been associated with long-term effects, such as mother's postpartum weight retention [7]; and higher risk of childhood obesity [8] or neurodevelopmental impairment in children [9]. Conversely, insufficient GWG has been related with preterm birth [6].

Offspring' cognitive skills have been related to mental and physical health $[10,11]$, and some observational studies have reported positive, negative or null association between GWG as exposure and neurodevelopment in childhood and adolescence [9, 12-17]. This fact could be due offspring's neurodevelopment is associated with several factors throughout the different stages of life [18]. During antenatal period, folic acid supplementation could have a positive effect on cognitive development, [19] as well as the treatment with magnesium sulfate or corticosteroids in preterm births that could prevent neurodevelopmental delay. Furthermore, breastfeeding [20, 21], physical activity, [22] education [23] and a good home environment $[24,25]$ could improve cognitive development. However, iron deficiency during childhood, $[18,26]$ lower maternal cognition test scores or low socioeconomic status have been associated with worse cognitive development [25].

Considering the potential effect of GWG on offspring's cognition, it seems necessary to examine the evidence on the association between GWG and children's cognitive development. Thus, our systematic review and meta-analysis aim to synthesize the evidence regarding the association between GWG and offspring's cognitive skills, distinguishing among intelligence quotient (IQ), language and mathematics-related skills.

\section{Methods}

This systematic review and meta-analysis was reported according to the Cochrane Collaboration Handbook recommendations [27] and Preferred Reporting Items for Systematics Reviews and Meta-Analysis PRISMA [28]. It has been registered in PROSPERO (Registration number: CRD42017073266).

\section{Search strategy}

Studies were identified through the following databases: MEDLINE, Web of Science, Scopus and Cochrane Library, from their inception to April 2020 without language restriction (Fig. 1). The search strategy included a combination of the following terms: (i) population: "pregnancy", "gestational maternal", "antepartum", "prenatal"; (ii) exposure: "weight gain", "weight change", "obesity"; (iii) cognitive skills: "academic achievement", "academic grades", "academic behaviour", "academic behavior", "academic performance", "academic", "attention", "classroom behaviour", "classroom behavior", cognition, "cognitive development", "cognitive function", "cognitive control", "cognitive achievement", "executive", "executive function", "intellectual", "intelligence", "neurodevelopment", "memory", "metacognition"; (iv) population: "birth", "infant", “child", "childhood", "children", "offspring", "adolescence" (Table S1). The search was manually managed for each database and the references of the selected studies were reviewed to identify additional studies.

\section{Inclusion criteria}

We included studies examining the association between GWG and offspring's cognitive skills if they met the following inclusion criteria: (i) outcomes: offspring's cognition assessed by standardized test scores or academic achievement; (ii) study design: observational or experimental studies; (iii) exposure: GWG; (iv) participants: mothers and their offspring without age restriction. Studies were excluded when they were focused exclusively on: (i) mothers with intellectual disabilities; (ii) mothers with diabetes, preeclampsia or cardiovascular problems; (iii) offspring with developmental disorders/ diagnoses like symptoms of autism spectrum disorder, because studies in which all children are affected with any detected delay in communication, adaptation, cognition or socio-emotional domains could modify the effect of the main exposure, GWG, on offspring's cognition and therefore might bias the estimates of meta-analysis.; and (iv) new-borns not born at full term (between 37 to 41 weeks of gestation).

The literature search was performed independently by two reviewers (J.A.M.H. and C.A.B.) and disagreements were solved by consensus. Both reviewers are health professionals and have extensive experience in systematic reviews and meta-analysis. The two reviewers independently examined the titles and abstracts of the studies. Full articles of all studies that appeared to fulfil the inclusion criteria or where there was some uncertainty were obtained for the two reviewers to independently 


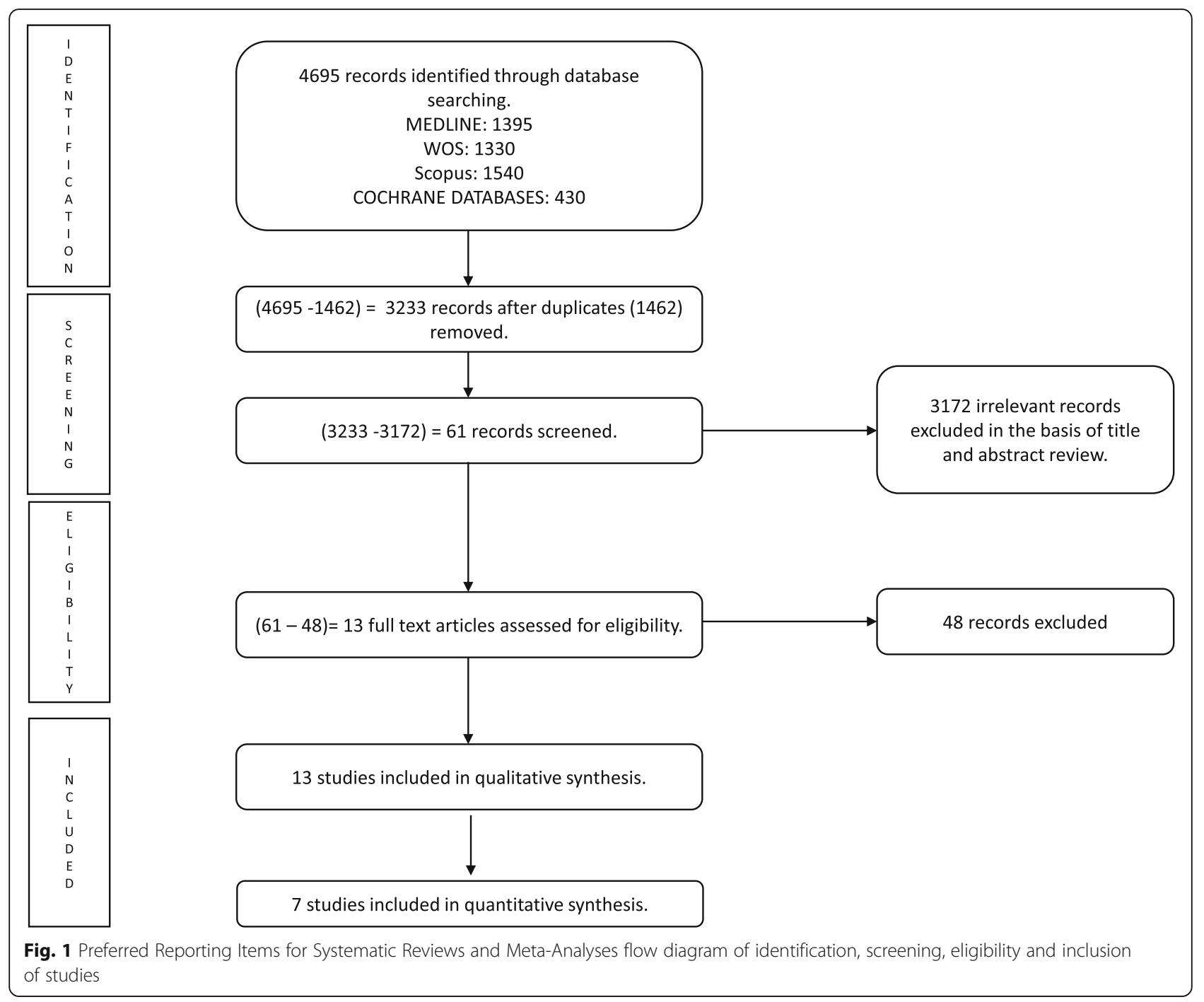

assess their eligibility. Studies that did not fulfil the selection criteria were excluded. The reviewers checked the included and excluded studies and verified the reasons for inclusion/exclusion. Any discrepancies were resolved by consensus and arbitration by a third reviewer (V.M.V.) that is health professional and has extensive experience in systematic reviews and meta-analysis. Reasons for exclusion were coded for both the initial assessment phase and the review phase of full text articles. The PRISMA flowchart will be used to document the study selection process. Kappa interrater agreement was 0.90 .

\section{Data extraction and quality reviews process}

The following data were extracted from the original reports: (i) study data (author, year of publication, country, year of birth, sample size of mothers and offspring, GWG classification criteria); (ii) characteristics of participants (offspring's age at evaluation and maternal variables: age at delivery, pre-pregnancy BMI and GWG); (iii) tools and/or scales used for assessing offspring's cognitive skills, (iv) cognition domains evaluated and (v) adjustment variables. Duplicated publications were identified based on the data extracted, when multiple articles from a study were identified on the same exposure and outcome variables, only the report with the largest sample size was included; However, if the study samples were independent or they reported different cognitive skills, they were treated as separate studies. If we needed additional information or clarification about any study, authors were contacted.

Data extraction was independently performed by two reviewers (J.A.M.H. and C.A.B.), and inconsistencies were solved by consensus. A third researcher was asked when consensus was not reached (V.M.V.).

Since no experimental study was retrieved, the Quality of Reporting of Observational Longitudinal Research scale was used to evaluate the risk of bias [29]. This 
rating list consists of 33 items and each criterion was assessed as "yes" (=1), "no" (=0) or "not applicable" (=?), resulting in a total score that ranges from 0 to 33 .

Quality assessment was independently performed by two reviewers (J.A.M.H. and C.A.B.), and inconsistencies were solved by consensus. A third researcher was asked when consensus was not reached (V.M.V.).

\section{Analysis}

Effect size (ES) and their respective 95\% confidence intervals $(95 \% \mathrm{CI})$ were used to examine the association between excessive or insufficient GWG and offspring's cognition. We considered three cognitive domains: IQ, language-related skills and mathematics-related skills. A standardized mean difference score was calculated for each pre-pregnancy weight status category as an estimate of ES [30]. When studies provided a linear regression $\beta$ coefficient, it was used to calculate a standardized mean difference score $[30,31]$. If a study reported stratified data, we performed a pooled estimation to unify data, for example when a study provided data by trimester of pregnancy, we combined data to obtain pooled results throughout pregnancy. The Mantel-Haenszel fixedeffect method [32] was used to compute pooled ES estimates and their respective 95\% CI, which were used to examine the association between GWG above or below recommendations and offspring's cognition, using GWG within recommendations as reference. In the case that one study reported more than one assessment of the same cognition domain at different ages we pooled them; we also polled the estimates of the same domain of cognition measured using different instruments. In the forest plots used to depict the ES of each study and the pooled ES estimates, a negative ES value indicated lower cognitive skills scores in offspring whose mothers had not recommended GWG as compared with offspring whose mothers had GWG within recommendations. The heterogeneity of results across studies was assessed by $\mathrm{I}^{2}$ statistic and it was classified as: not important (0 to $30 \%)$; moderate (30 to $50 \%$ ); substantial (50 to $75 \%$ ) and considerable (75 to $100 \%)$, also the corresponding $p$-values were considered [33].

For the analyses, we used: (i) the most adjusted models reported by included studies; (ii) GWG classification criteria published in 1990 by The National Academy of Science or own criteria stablished by original articles were considered similar to those of 2009 IOM recommendations. Furthermore, we conducted subgroup analyses by GWG classification (one group with 2009 IOM's guidelines and other group with the rest classifications) and by age (stratified as pre-schoolers, from 2 to 6 years old, and schoolers, between 6 and 12 years old).
Sensitivity analyses was conducted by removing studies one by one in order to evaluate the robustness of the summary estimates.

Finally, publication bias was evaluated by visual inspection of funnel plot, and according to the method proposed by Egger [34]. Statistical analyses were performed using StataSE 15.

\section{Results}

From 4498 articles retrieved through the systematic search, 61 were eligible for full-text assessment. Finally, 13 studies were included in the qualitative synthesis [9, 12-17, 35-40] and seven in the quantitative synthesis (Fig. 1) [13, 14, 17, 35, 38-40].

We only included 7 studies in quantitative synthesis for some reasons $[13,14,17,35,38-40]$ : one study did not provide a reference group of GWG to compare the association between GWG and offspring's cognition [12]; three did not provide quantitative data of offspring's cognitive skills that we studied [9, 16, 36]; and three studies shared the same sample and we only included them in the same pooled when assessed different domain of cognition $[9,12,14]$.

\section{Study characteristics}

Table 1 summarizes the main characteristics of the included studies. Included studies were published between 1981 and 2018. Ten studies were conducted in the United States [9, 12, 14, 16, 17, 35-37, 39, 40], one in Norway and Sweden [13], United Kingdom [38] and China [15]. All are cohort studies: eight studies reported data for one follow-up evaluation $[9,13,15-17,36,37$, 40], two studies for two follow-up evaluations [12, 14, 39] and two studies provided data for three follow-up evaluations [35, 38].

The included sample size ranged from 355 to 31,968 offspring whose year of birth ranged from 1959 to 2009. The age at which offspring's cognitive skills were assessed varied from 8 months to 16 years. The average mother's age ranged from $21.3^{9}$ to 29.1 years [13].

Seven studies used the IOM recommendations published in 2009 to classify GWG [9, 13, 14, 17, 37-39], one study used those published in 1990 [16], four studies used their own reference values to establish insufficient or excessive GWG $[12,35,36,40]$ and one did not report any classification [15].

\section{Cognitive skills assessed}

The cognitive domains assessed in included studies were: (i) IQ [6, 9, 13, 14, 35, 37, 38], (ii) language-related skills, [13, 14, 16, 17, 35, 37] and (iii) mathematicsrelated skills $[14,17,35]$. Other cognitive domains included in the studies were: (i) general intelligence [16, 39], (ii) executive function [12, 16, 40], (iii) non-verbal 
Martínez-Hortelano et al. BMC Pediatrics $\quad$ (2020) 20:533

Page 5 of 12

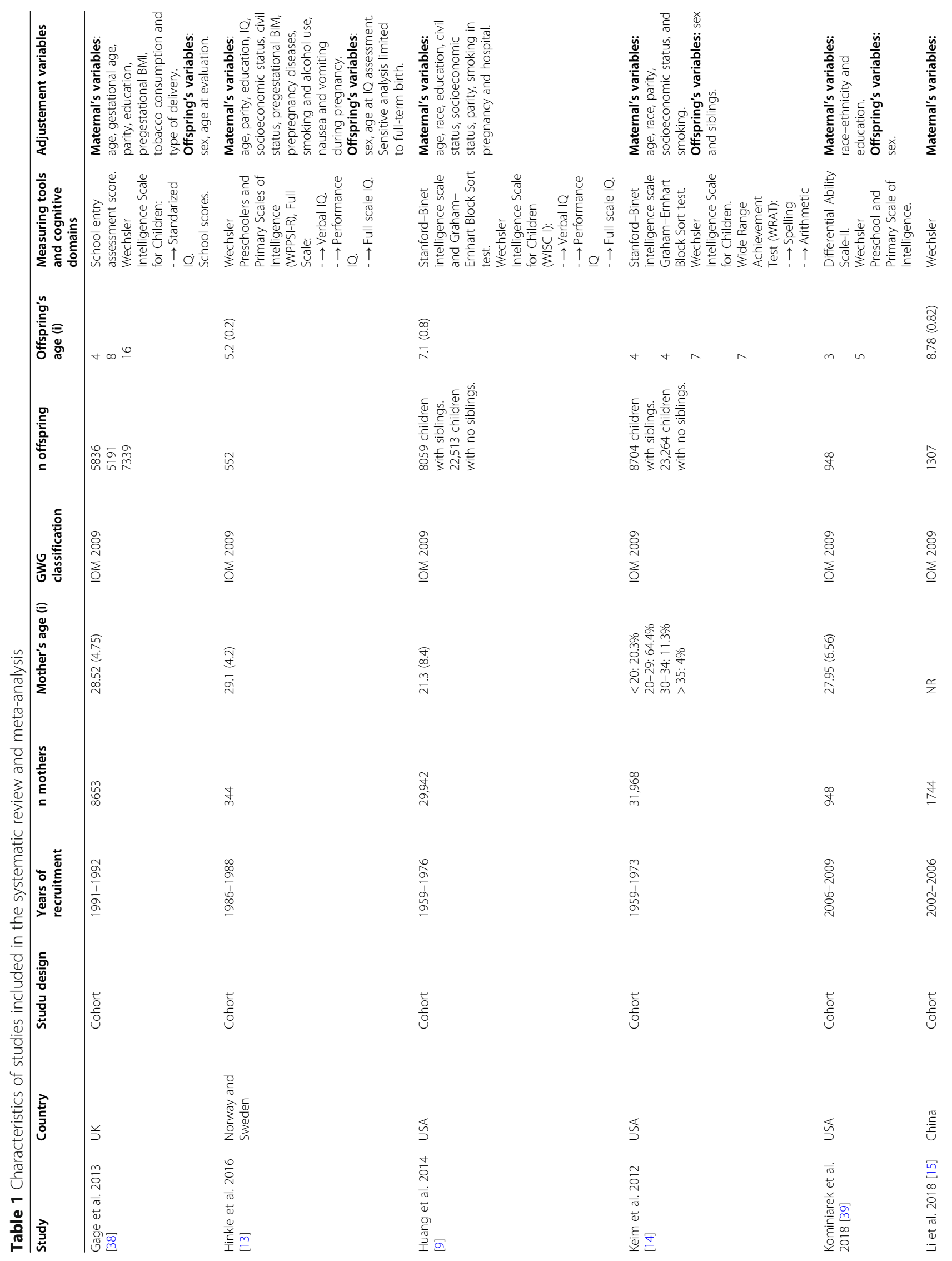




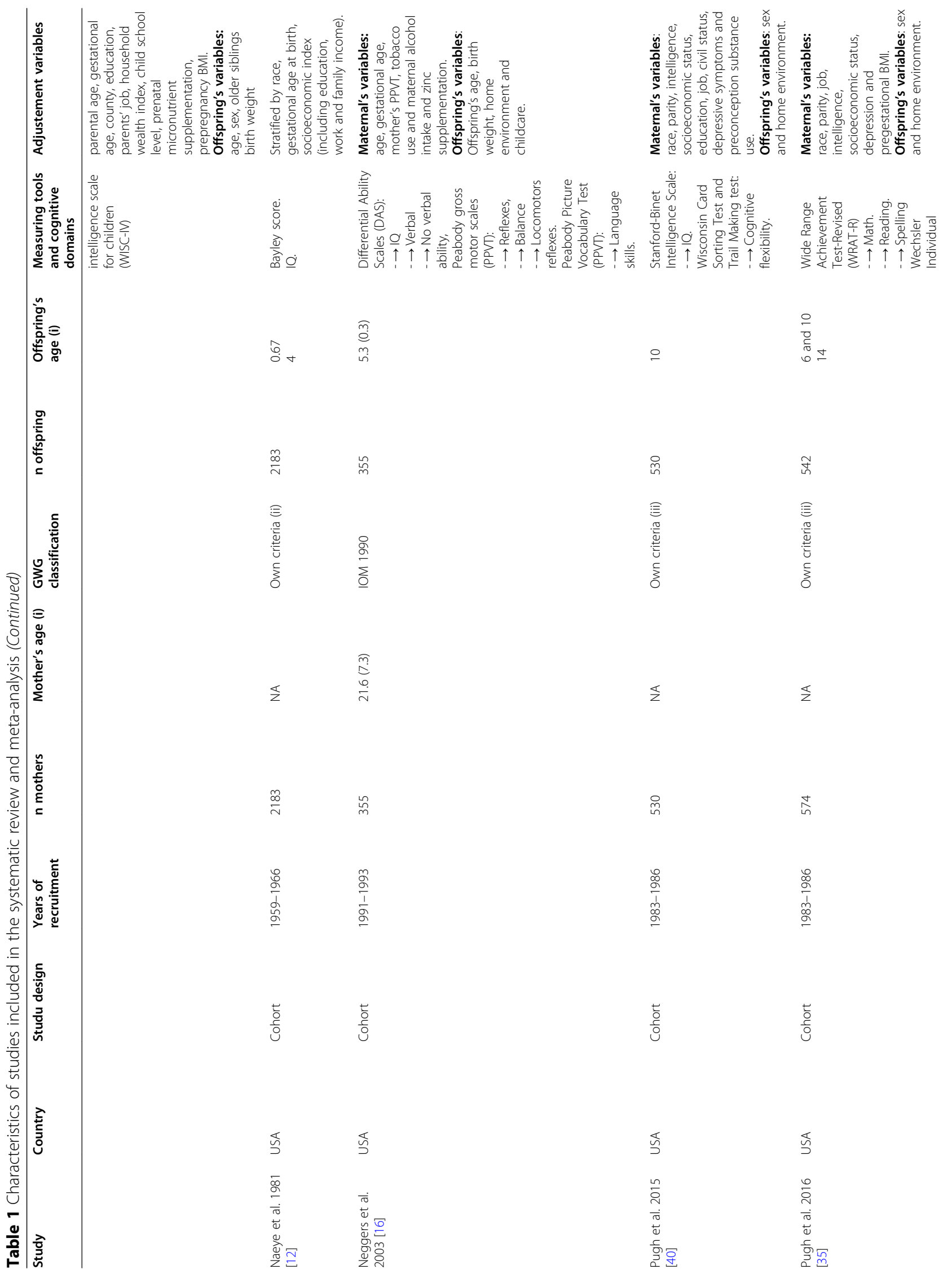




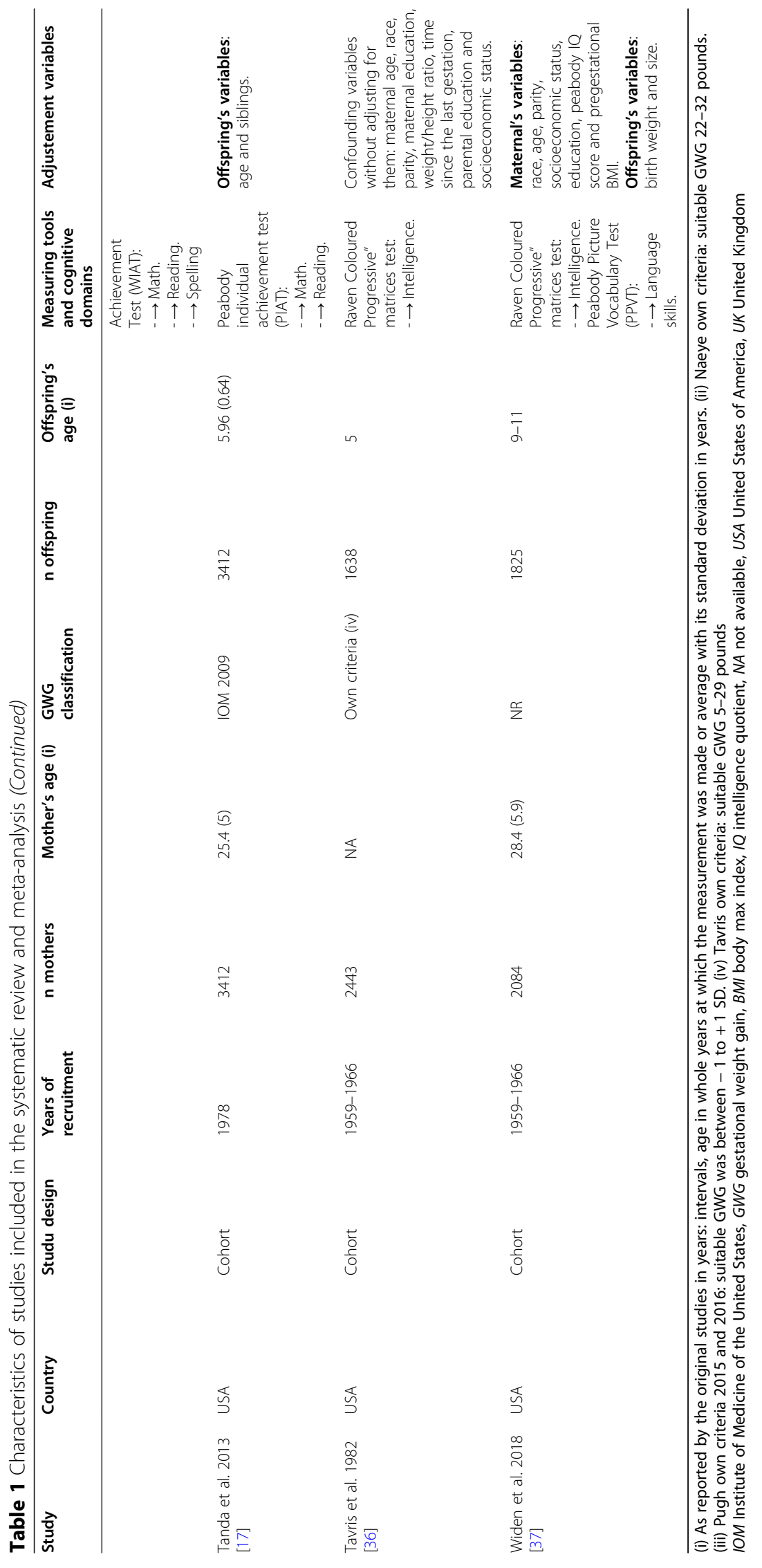


skills $[16,40]$, and (iv) total scores in academic achievement [38]. Tables S2 to S5 summarize the association between GWG and offspring's cognitive skills as reported in the original articles and their ES.

The most common variables used in the adjusted analyses were: (i) maternal covariates such as maternal age, pre-pregnancy BMI, parity, race, intelligence, education or gestational age at delivery; (ii) family background covariates including marital status, socioeconomic status or home environment; and (iii) offspring's covariates such as sex and age. Only two studies did not report adjusted models $[12,36]$ and three did not report quantitative data of the association between GWG and offspring's cognitive skills [9, 16]. Among the most important variables that could affect the relationship between GWG and offspring's cognition between studies included in the metaanalysis: (i) gestational age at birth was taken into account for almost all studies in different ways (only included fullterm pregnancies [14, 17, 38-40], adjusted by gestational age [35] or conducted a sensitivity analysis) [13]; (ii) one study only included women with a normal pre-pregnancy BMI [40], and three adjusted by pre-pregnancy BMI [13, 35, 38]; (iii) postnatal environment was taken into account in all studies as different variables.

\section{Meta-analysis}

The ES for the association of GWG above recommendations with offspring's IQ was 0.02 (95\% CI -0.00, 0.05; $\left.\mathrm{I}^{2}=0.00 \%\right)$, language related skills 0.00 (95\% CI -0.05 , $\left.0.05 ; \mathrm{I}^{2}=0.00 \%\right)$ and mathematics related skills 0.01 (95\% CI -0.01, 0.04; $\mathrm{I}^{2}=0.00 \%$ ) (Fig. 2).

Furthermore, the ES for the association of GWG below recommendations with offspring's IQ was 0.00 (95\% CI $\left.-0.02,0.03 ; \mathrm{I}^{2}=0.00 \%\right)$, language-related skills $0.02(95 \%$ CI $\left.-0.03,0.05 ; \mathrm{I}^{2}=0.00 \%\right)$ and mathematics-related skills 0.01 (95\% CI: - 0.04, 0.05; $\mathrm{I}^{2}=0.00 \%$ ) (Fig. 3).

Other cognitive domains were not included in the meta-analysis, but these studies did not find association between GWG and general intelligence [16, 39], executive functions $[12,16,39,40]$ non-verbal skills $[16,40]$ or short term memory [40]. However, GWG was positively associated with better academic achievement at 16 years of age [38].

\section{Subgroup analyses}

We did not observe differences by GWG classification or age when we conducted subgroup analyses (Tables S6 to $\mathrm{S} 17$ ).

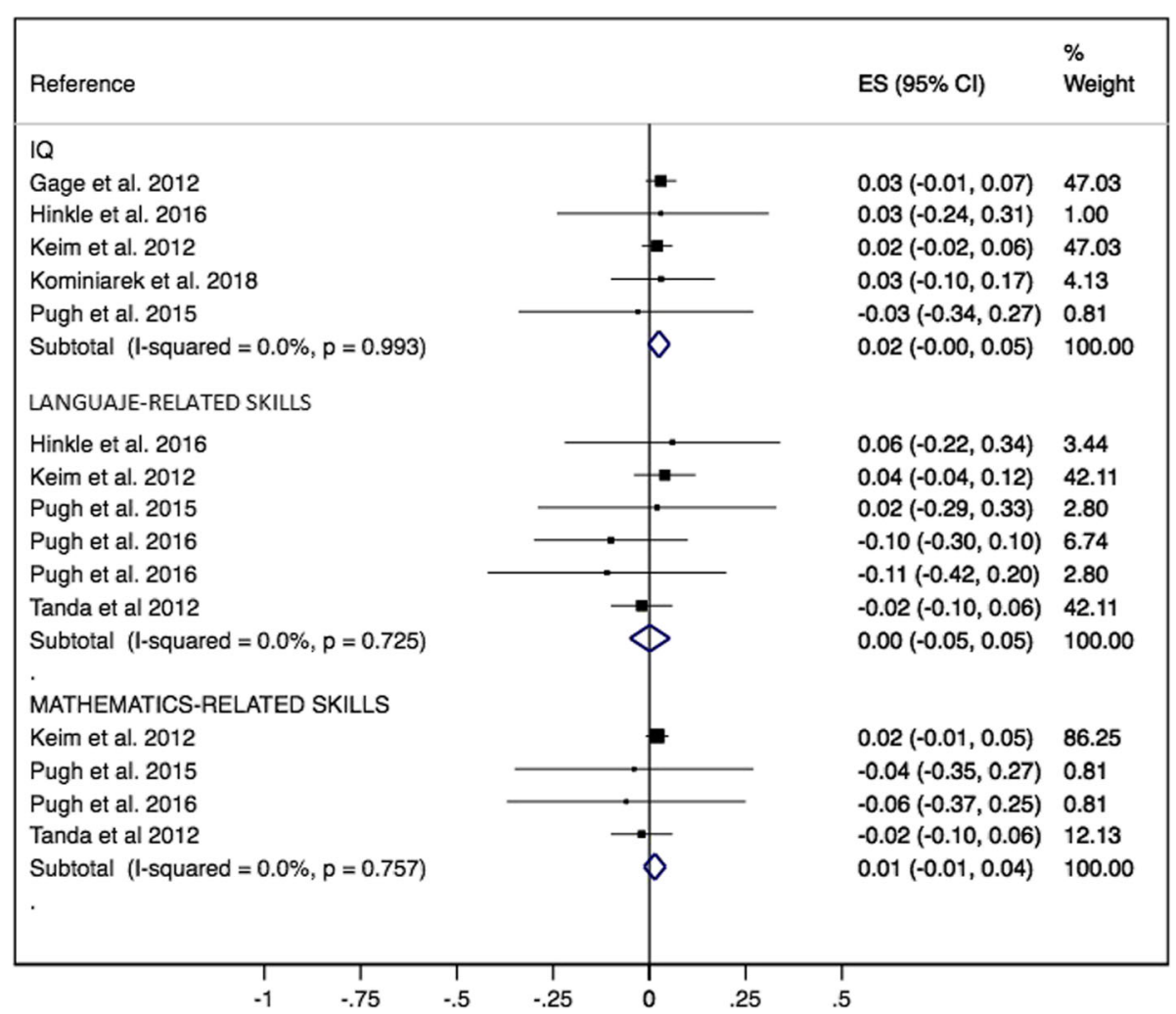

Fig. 2 Offspring's intelligence quotient, language related skills and mathematics related skills forest plot whose mother's had GWG above recommendations comparing with GWG within recommendations 


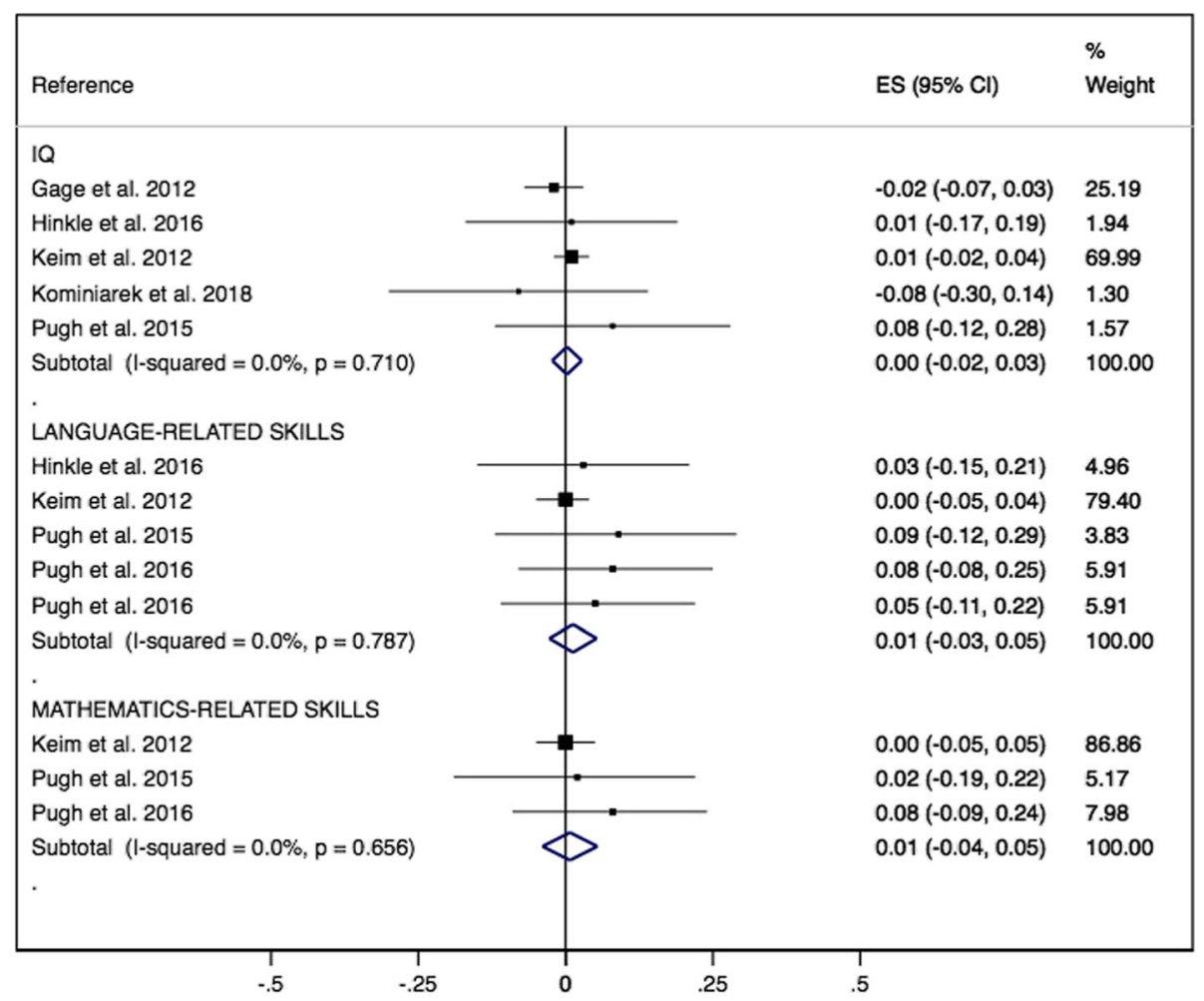

Fig. 3 Offspring's intelligence quotient, language related skills and mathematics related skills forest plot whose mother's had GWG below recommendations comparing with GWG within recommendations

\section{Sensitivity analyses.}

Sensitivity analyses suggested that the pooled ES or the heterogeneity were not modified in any model after removing the included studies one by one (Tables S18 to S23).

\section{Assessment of publication Bias.}

Funnel plots appear symmetrical and the Egger test revealed that there was no evidence of publication bias in any model ( $p$ values higher than 0.1 ), except for the model comparing offspring's language related skills between mothers who had GWG below recommendation versus mothers who had GWG within recommendations $(p=0.02)$.

\section{Quality of reporting}

The level of compliance for the risk of bias assessment varied from 55 to $91 \%$ (Table S24). None of the studies justified the number of participants. Furthermore, four studies reported a quantitative statement of consenters or non-consenters $[13,16,35,40]$ and four reported reasons for loss to follow up $[15,17,36,40]$. Regarding statistical analysis methods, one study took into account loss to follow up [13] and two considered missing data in the analysis $[13,40]$. Finally, one reported the impact of biases estimated quantitatively [17].

\section{Discussion}

The findings of this systematic review and meta-analysis support that the weight gain during pregnancy above recommendations could have a small positive influence in offspring's IQ, but the expected relationship between GWG above recommendations and language or mathematics related skills was not confirmed. Finally, GWG below the recommendations was not associated neither with IQ, nor with language and mathematics related skills.

Our pooled estimates found a small positive relationship between GWG and offspring's IQ, although not statistically significant, in line with a previous study that reported a positive linear association between GWG until 28 weeks and offspring's IQ [38]. This fact could be explained because greater GWG may produce greater maternal fat deposition, and might result in a greater delivery of glucose and fatty acids, which could positively influence the fetal brain development [38]. The results of the included studies are inconsistent, while some studies reported association between GWG and offspring's cognitive skills $[9,14,38]$, others did not provide any association $[12-17,35,37,40]$. To explain these mixed results, previous published articles reported biological mechanisms that could underlying the potential relationships. For example, an increased inflammatory 
markers level as a result of excessive maternal fat deposition, which might contribute to an adverse environment for fetal brain development [41] and epigenetic alterations [42-44]. In contrast, synapse formation and myelination are vulnerable to undernutrition, hence insufficient GWG could be associated with suboptimal neurodevelopment [45]. All studies reported weak associations between GWG and offspring's cognitive skills and this fact could be due to the exposure of GWG is limited by pregnancy period and offspring's cognitive skills are influenced by other postnatal factors that could dilute the effect of perinatal factors in older offspring such as home environment or physical activity during childhood $[46,47]$. The magnitude of the association between GWG and offspring's cognitive skills is similar to those reported for other exposures around pregnancy and lactation periods, such as pre-pregnancy BMI or breast feeding [48, 49].

Several reasons can be adduced to explain variability across studies and differences between adjusted and unadjusted models found in included studies [13, 38] may be due to the variability in the covariates included in the models. Obesity before pregnancy have a negative influence on offspring's cognitive skills [48] and it is possible that GWG within recommendations might mitigate this negative association [9], although the pathways behind this relationship are not entirely clear [38, 41, 42, 45]. Not only pre-pregnancy obesity, but also other perinatal and postnatal exposures could in greater or lesser extend to influence cognitive development [47, 50-52]. Thus, two studies proposed a novel analysis by siblings to control for observed variables or unobserved factors that are shared by siblings, because they account for all genetic and environmental factors in common $[9,14]$.

\section{Strengths and limitations}

The large total sample size accumulated by included studies, the lack of publication bias (except for the model that compared offspring's language related skills between mothers who had GWG below recommendation versus mothers who had GWG within recommendations.), the standardized tests used to assess cognitive skills were according to offspring's age and the consistency of results proven in sensitivity analyses are among the strengths of this study.

However, some limitations should also be acknowledged in this article: (i) the offspring's cognitive measuring tools were different in each study; (ii) some studies included subsamples of disadvantaged subgroups or population subgroups, which could influence the magnitude of the associations; (iii) cohorts were living in countries where the health related behaviours are presumably different, which could influence cognitive development, although this could be mitigate in the adjusted model $[9,14,16$, 36]; (iv) we could not performed a subgroup analyses by pre-pregnancy BMI, gestational age at birth, offspring's age at cognitive assessment or trimester specific because there were few studies that reported these subgroup analyses; (v) studies included used different criteria for GWG classification; (vi) the lack of trials make impossible to stablish causality between GWG and offspring's cognition.

\section{Implication for research}

The GWG should be appropriately manage by clinicians for improving neonatal and maternal outcomes, among them offspring's cognitive skills. Thus, prenatal care providers, who are in a privileged position to manage GWG, should give individualized advice on physical activity and diet to women to prioritize an adequate or a careful excessive GWG because it could improve offspring's IQ according to our findings [53-57].

Due to the variability reported by original articles and the differences in sample characteristics across studies and study designs we highlight in the importance considerations for future research: (i) the use of 2009 IOM's guidelines to classify GWG, (ii) the use of validated tests to assess cognitive skills and (iii) if sibling models are not possible, the control of potential confounders in the analysis $[9,14]$.

\section{Conclusion}

Our systematic review and meta-analysis supports that GWG above recommendations could improve offspring's IQ. However, we found no association between GWG and offspring's cognitive skills, although differences in the sample characteristics and analyses may explain the variability in results reported by the original articles. The generalizability of our results could be limited due to the lack of homogeneous design of included studies, but our findings could provide an initial approach for elucidating the association between GWG and offspring's cognition. Potential influence of perinatal and postnatal variables could be behind this inconsistence. Further high-quality studies are needed including population-based samples, using the same GWG classification criteria and validated offspring's cognitive assessment tools.

\section{Supplementary Information}

The online version contains supplementary material available at https://doi. org/10.1186/s12887-020-02429-7.

Additional file 1: Table S1. Search strategy for MEDLINE database.

Additional file 2: Tables S2 to S5. Calculations.

Additional file 3: Tables S6 to $\mathbf{S 1 7}$. Subgroup analyses by GWG classification. Tables $\mathbf{S 1 8}$ to $\mathbf{S 2 3}$. Sensitivity analyses.

Additional file 4: Table S24. Quality assessment by Quality of reporting of observational longitudinal research scale.

\section{Abbreviations}

GWG: Gestational weight gain; IOM: Institute of Medicine; BMI: Body mass index; ES: Effect size; IQ: Intelligence quotient 


\section{Acknowledgements}

None.

\section{Authors' contributions}

J.A.M.H. and C.A.B. conceived the idea for the review, conducted the database searches, selected and retrieved relevant papers, designed the data extraction tool, carried out data extraction, checking of data, and quality assessments, and wrote the article. ICR-contributed to the design of the study, the analysis and interpretation, carried out extraction/checking of data and quality assessments. C.B.M. and A.H.A. reviewed and edited the article and contributed to the checking of data and quality assessment. V.M.V. academic achievement contributed to the study conception, made the final decisions regarding the inclusion/exclusion of all papers, and reviewed and edited the article. All authors have read and approved the manuscript.

\section{Funding}

This study did not receive any funding.

\section{Availability of data and materials}

Supporting data can be obtained from the corresponding author.

\section{Ethics approval and consent to participate}

Not applicable.

\section{Consent for publication}

Not applicable.

\section{Competing interests}

Authors declare that they have no competing interests.

\section{Author details}

'Universidad de Castilla-La Mancha, Centro de Estudios Sociosanitarios, Cuenca, Spain. ${ }^{2}$ Universidad Politécnica y Artística del Paraguay, Asunción, Paraguay. ${ }^{3}$ Universidad Autónoma de Chile, Facultad de Ciencias de la Salud, Talca, Chile.

Received: 23 April 2020 Accepted: 13 November 2020

Published online: 26 November 2020

\section{References}

1. Institute of Medicine. Nutrition During Pregnancy: Part I: Weight Gain, Part II: Nutrient Supplements. Washington, DC: The National Academies Press. 1990. https://doi.org/10.17226/1451

2. Institute of Medicine. Weight Gain During Pregnancy. Washington, D.C: National Academies Press; 2009. https://doi.org/10.17226/12584.

3. Ferrari N, Mallmann P, Brockmeier K, Strüder HK, Graf C. Secular trends in pregnancy weight gain in German women and their influences on foetal outcome: a hospital-based study. BMC Pregnancy Childbirth. 2014;14(1):228. https://doi.org/10.1186/1471-2393-14-228.

4. Goldstein RF, Abell SK, Ranasinha S, et al. Gestational weight gain across continents and ethnicity: systematic review and meta-analysis of maternal and infant outcomes in more than one million women. BMC Med. 2018; 16(1):153. https://doi.org/10.1186/s12916-018-1128-1.

5. Johnson JL, Farr SL, Dietz PM, Sharma AJ, Barfield WD, Robbins CL. Trends in gestational weight gain: the Pregnancy Risk Assessment Monitoring System, 2000-2009. Am J Obstet Gynecol. 2015;212(6):806.e1-8. https://doi.org/10. 1016/j.ajog.2015.01.030

6. Kominiarek MA, Saade G, Mele L, et al. Association between gestational weight gain and perinatal outcomes. Obstet Gynecol. 2018;132(4):875-81. https://doi.org/10.1097/AOG.0000000000002854.

7. Nehring I, Schmoll S, Beyerlein A, Hauner H, von Kries RR. Gestational weight gain and long-term postpartum weight retention: a meta-analysis. Am J Clin Nutr. 2011;94(5):1225-31. https://doi.org/10.3945/ajcn.111.015289.

8. Sridhar SB, Darbinian J, Ehrlich SF, et al. Maternal gestational weight gain and offspring risk for childhood overweight or obesity. Am J Obstetr Gynecol. 2014;211:259.e1-8. https://doi.org/10.1016/j.ajog.2014.02.030.

9. Huang L, Yu X, Keim S, Li L, Zhang L, Zhang J. Maternal prepregnancy obesity and child neurodevelopment in the collaborative perinatal project. Int J Epidemiol. 2014;43(3):783-92. https://doi.org/10.1093/ije/dyu030.
10. Koenen KC, Moffitt TE, Roberts AL, et al. Childhood IQ and adult mental disorders: a test of the cognitive reserve hypothesis. Am J Psychiatry. 2009; 166(1):50-7. https://doi.org/10.1176/appi.ajp.2008.08030343.

11. Martin LT, Kubzansky LD. Childhood cognitive performance and risk of mortality: a prospective cohort study of gifted individuals. Am J Epidemiol. 2005. https://doi.org/10.1093/aje/kwi300.

12. Naeye RL, Chez RA. Effects of maternal acetonuria and low pregnancy weight gain on children's psychomotor development. Am J Obstet Gynecol. 1981;139(2):189-93.

13. Hinkle SN, Albert PS, Sjaarda LA, Grewal J, Grantz KL. Trajectories of maternal gestational weight gain and child cognition assessed at 5 years of age in a prospective cohort study. J Epidemiol Community Health. 2016;70(7):696703. https://doi.org/10.1136/jech-2014-205108.

14. Keim SA, Pruitt NT. Gestational weight gain and child cognitive development. Int J Epidemiol. 2012;41(2):414-22. https://doi.org/10.1093/ije/ dyr229.

15. Li C, Zhu N, Zeng $L$, et al. Effect of maternal pre-pregnancy underweight and average gestational weight gain on physical growth and intellectual development of early school-aged children. Sci Rep. 2018;8(1):12014. https://doi.org/10.1038/s41598-018-30514-6.

16. Neggers YH, Goldenberg RL, Ramey SL, Cliver SP. Maternal prepregnancy body mass index and psychomotor development in children. Acta Obstet Gynecol Scand. 2003;82(3):235-40.

17. Tanda R, Salsberry PJ, Reagan PB, Fang MZ. The impact of prepregnancy obesity on children's cognitive test scores. Matern Child Health J. 2013;17(2): 222-9. https://doi.org/10.1007/s10995-012-0964-4.

18. Vaivada T, Gaffey MF, Bhutta ZA. Promoting early child development with interventions in health and nutrition: A systematic review. Pediatrics. 2017; 140(2). https://doi.org/10.1542/peds.2016-4308.

19. McNulty H, Rollins M, Cassidy T, et al. Effect of continued folic acid supplementation beyond the first trimester of pregnancy on cognitive performance in the child: A follow-up study from a randomized controlled trial (FASSTT Offspring Trial). BMC Med. 2019;17(1). https://doi.org/10.1186/ s12916-019-1432-4.

20. Drane $\mathrm{DL}$, Logemann JA. A critical evaluation of the evidence on the association between type of infant feeding and cognitive development. Paediatr Perinat Epidemiol. 2000;14(4):349-56 http://www.ncbi.nlm.nih.gov/ pubmed/11101022. Accessed 5 Feb 2019.

21. McCrory C, Murray A. The effect of breastfeeding on Neuro-development in infancy. Matern Child Health J. 2013;17(9):1680-8. https://doi.org/10.1007/ s10995-012-1182-9.

22. Sibley BA, Etnier JL. The relationship between physical activity and cognition in children: a meta-analysis. Pediatr Exerc Sci. 2003;15(3):243-56. https://doi.org/10.1123/pes.15.3.243

23. Burger K. How does early childhood care and education affect cognitive development? An international review of the effects of early interventions for children from different social backgrounds. Early Child Res Q. 2010;25(2): 140-65. https://doi.org/10.1016/j.ecresq.2009.11.001.

24. Crane J. Effects and of Home Maternal Test Environment, Scores on SES , Mathematics. J Educ Res. 1996;89(5):305-14. https://doi.org/10.2307/ 27542048

25. Feingold C. Correlates of cognitive development in low-birth-weight infants from low-income families. J Pediatr Nurs. 1994;9(2):91-7 http://www.ncbi. nlm.nih.gov/pubmed/7518000. Accessed 5 Feb 2019

26. Grantham-McGregor S, Ani C. A review of studies on the effect of iron deficiency on cognitive development in children. J Nutr. 2001. https://doi. org/10.1093/jn/131.2.649s.

27. Higgins J, Green S. Cochrane handbook for systematic reviews of interventions; 2005.

28. Moher D, Liberati A, Tetzlaff J, Altman DG, Group P. Preferred reporting items for systematic reviews and meta-analyses: the PRISMA statement. Int J Surg. 2010;8(5):336-41. https://doi.org/10.1016/j.ijsu.2010.02.007.

29. Tooth L, Ware R, Bain C, Purdie DM, Dobson A. Quality of reporting of observational longitudinal research. Am J Epidemiol. 2005;161(3):280-8. https://doi.org/10.1093/aje/kwi042.

30. Lipsey M, Wilson D. Practical meta-analysis; 2001

31. Peterson RA, Brown SP. On the use of beta coefficients in meta-analysis. J Appl Psychol. 2005:90(1):175-81. https://doi.org/10.1037/0021-9010.90.1.175.

32. MANTEL N, HAENSZEL W. Statistical aspects of the analysis of data from retrospective studies of disease. J Natl Cancer Inst. 1959:22(4):719-48 https://www.ncbi.nlm.nih.gov/pubmed/13655060. 
33. Deeks JJ, Higgins JP, Altman DG. Analysing Data and Undertaking MetaAnalyses. In: Cochrane Handbook for Systematic Reviews of Interventions. Chichester: Wiley. p. 243-96. https://doi.org/10.1002/9780470712184.ch9.

34. Egger M, Smith GD, Schneider M, et al. Bias in meta-analysis detected by a simple, graphical test. BMJ. 1997;315(7109):629-34. https://doi.org/10.1136/ BMJ.315.7109.629.

35. Pugh SJSJ, Hutcheon JAJA, Richardson GAGAGA, et al. Child academic achievement in association with pre-pregnancy obesity and gestational weight gain. J Epidemiol Community Health. 2016;70(6):534-40. https://doi. org/10.1136/jech-2015-206800.

36. Tavris DR, Read JA. Effect of maternal weight gain on fetal, infant, and childhood death and on cognitive development. Obstet Gynecol. 1982; 60(6):689-94.

37. Widen EM, Kahn LG, Cirillo P, Cohn B, Kezios KL, Factor-Litvak P. Prepregnancy overweight and obesity are associated with impaired child neurodevelopment. Matern Child Nutr. 2018;14(1):e12481. https://doi.org/10. 1111/mcn.12481

38. Gage SH, Lawlor DA, Tilling K, Fraser A. Associations of maternal weight gain in pregnancy with offspring cognition in childhood and adolescence: findings from the Avon longitudinal study of parents and children. Am J Epidemiol. 2013;177(5):402-10. https://doi.org/10.1093/aje/kws239.

39. Kominiarek MA, Smid MC, Mele L, et al. Child neurodevelopmental outcomes by Prepregnancy body mass index and gestational weight gain. Obstet Gynecol. 2018;132(6):1. https://doi.org/10.1097/AOG. 0000000000002974.

40. Pugh SJ, Richardson GA, Hutcheon JA, et al. Maternal obesity and excessive gestational weight gain are associated with components of child cognition. J Nutr. 2015;145(11):2562-9. https://doi.org/10.3945/jn.115.215525.

41. van der Burg JW, Sen S, Chomitz VR, Seidell JC, Leviton A, Dammann O. The role of systemic inflammation linking maternal BMI to neurodevelopment in children. Pediatr Res. 2016;79(1-1):3-12. https://doi.org/10.1038/pr.2015.179.

42. Berger SL, Kouzarides T, Shiekhattar R, Shilatifard A. An operational definition of epigenetics. Genes Dev. 2009;23(7):781-3. https://doi.org/10. 1101/gad.1787609.

43. Brion M-JJ, Zeegers $M$, Jaddoe $V$, et al. Intrauterine effects of maternal prepregnancy overweight on child cognition and behavior in 2 cohorts. Pediatrics. 2011;127(1):e202-11. https://doi.org/10.1542/peds.2010-0651.

44. Rodriguez A, Miettunen J, Henriksen TB, et al. Maternal adiposity prior to pregnancy is associated with ADHD symptoms in offspring: evidence from three prospective pregnancy cohorts. Int J Obes. 2008;32(3):550-7. https:// doi.org/10.1038/sj.ijo.0803741.

45. Georgieff MK. Nutrition and the developing brain: nutrient priorities and measurement. Am J Clin Nutr. 2007;85(2):614S-20S. https://doi.org/10.1093/ ajcn/85.2.614S

46. Donnelly JE, Lambourne K. Classroom-based physical activity, cognition, and academic achievement. Prev Med. 2011;52(SUPPL). https://doi.org/10.1016/j. ypmed.2011.01.021.

47. Tong S, Baghurst P, Vimpani G, McMichael A. Socioeconomic position, maternal IQ, home environment, and cognitive development. J Pediatr. 2007;151(3):284-288, 288.e1. https://doi.org/10.1016/j.jpeds.2007.03.020.

48. Álvarez-Bueno C, Cavero-Redondo I, Lucas-de la Cruz L, Notario-Pacheco B, Martínez-Vizcaíno V. Association between pre-pregnancy overweight and obesity and children's neurocognitive development: a systematic review and meta-analysis of observational studies. Int J Epidemiol. 2017;46(5):165366. https://doi.org/10.1093/ije/dyx122.

49. Kramer MS, Aboud F, Mironova E, et al. Breastfeeding and child cognitive development. Arch Gen Psychiatry. 2008;65(5):578. https://doi.org/10.1001/ archpsyc.65.5.578.

50. Camprubi Robles M, Campoy C, Garcia Fernandez L, Lopez-Pedrosa JM, Rueda R, Martin MJ. Maternal Diabetes and Cognitive Performance in the Offspring: A Systematic Review and Meta-Analysis. Meyre D, ed. Plos One. 2015;10(11):e0142583. https://doi.org/10.1371/journal.pone.0142583.

51. Heikura U, Hartikainen A-LL, Nordström T, Pouta A, Taanila A, Järvelin M-RR. Maternal hypertensive disorders during pregnancy and mild cognitive limitations in the offspring. Paediatr Perinat Epidemiol. 2013;27(2):188-98. https://doi.org/10.1111/ppe.12028.

52. Yang S, Platt RW, Kramer MS. Variation in child cognitive ability by week of gestation among healthy term births. Am J Epidemiol. 2010;171(4):399-406. https://doi.org/10.1093/aje/kwp413.

53. Sanabria-Martínez G, García-Hermoso A, Poyatos-León R, González-García A, Sánchez-López M, Martínez-Vizcaíno V. Effects of exercise-based interventions on neonatal outcomes. Am J Health Promot. 2016;30(4):21423. https://doi.org/10.1177/0890117116639569.

54. Poyatos-León R, García-Hermoso A, Sanabria-Martínez G, Álvarez-Bueno C, Sánchez-López M, Martínez-Vizcaíno V. Effects of exercise during pregnancy on mode of delivery: a meta-analysis. Acta Obs Gynecol Scand. 2015;94(10): 1039-47. https://doi.org/10.1111/aogs.12675.

55. Muktabhant B, Lawrie TA, Lumbiganon P, Laopaiboon M. Diet or exercise, or both, for preventing excessive weight gain in pregnancy. Cochrane Database Syst Rev. 2015;6:CD007145. https://doi.org/10.1002/14651858. CD007145.pub3.

56. Borge TC, Aase H, Brantsæter AL, Biele G. The importance of maternal diet quality during pregnancy on cognitive and behavioural outcomes in children: A systematic review and meta-analysis. BMJ Open. 2017;7(9). https://doi.org/10.1136/bmjopen-2017-016777.

57. Robinson AM, Bucci DJ. Maternal Exercise and Cognitive Functions of the Offspring. Cogn Sci. 7(2):187-205 http://www.ncbi.nlm.nih.gov/ pubmed/26664667. Accessed 22 Jan 2020.

\section{Publisher's Note}

Springer Nature remains neutral with regard to jurisdictional claims in published maps and institutional affiliations.

\section{Ready to submit your research? Choose BMC and benefit from:}

- fast, convenient online submission

- thorough peer review by experienced researchers in your field

- rapid publication on acceptance

- support for research data, including large and complex data types

- gold Open Access which fosters wider collaboration and increased citations

- maximum visibility for your research: over $100 \mathrm{M}$ website views per year

At BMC, research is always in progress.

Learn more biomedcentral.com/submissions 